\title{
On the Design and Development of a Zigbee-Based Multimodal Input-Output Monitoring-Actuating System
}

\author{
A. Orand*, Y. Tomita*, S. Okamoto**, S. Sonoda*** \\ * Department of Rehabilitation, Fujita Memorial Nanakuri Institute, Fujita Health University, Hisai-Isshiki-cho \\ 1865,Tsu, Mie 514-1296, Japan, Tel: +81 059-252-2744, Fax: +81 059-252-0710 \\ ** Department of Rehabilitation Medicine II, School of Medicine, Fujita Health University, 424-1 Oodoricho, Tsu, Mie \\ 514-1295, Japan, Tel: +81 059-252-2744, Fax: +81 059-252-0710
}

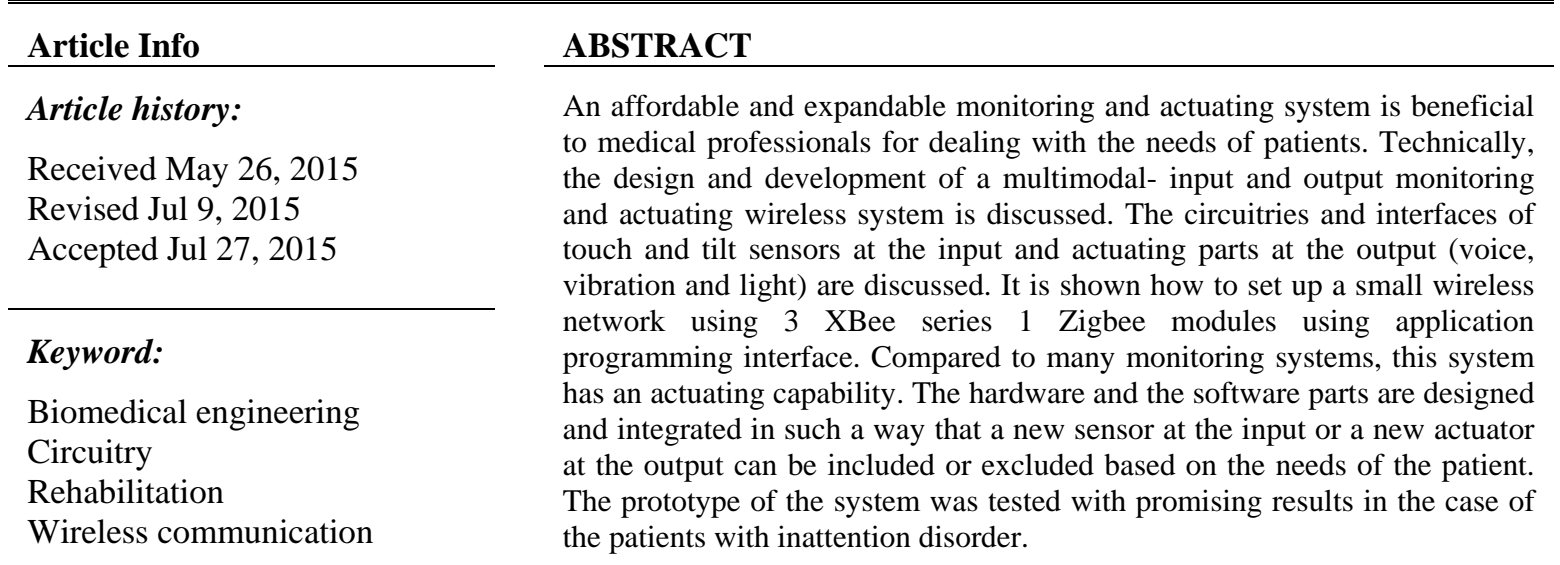

Copyright () 2015 Institute of Advanced Engineering and Science. All rights reserved.

\section{Corresponding Author:}

A. Orand, Department of Rehabilitation, Fujita Memorial Nanakuri Institute, Fujita Health University, Hisai-Isshikicho 1865,Tsu, Mie 514-1296, Japan, Tel: +81 059-252-2744, Fax: +81 059-252-0710

Email: orand@fujita-hu.ac.jp

\section{INTRODUCTION}

The principles of engineering have been applied to the fields of medicine [1, 2] and rehabilitation to advance health care treatment. Such assistive and assessing/alarming devices are applied to the hemiplegic patients who suffer from higher cortical function disorder and inattention disorder. Such studies include the application of vestibular stimulation [3], visual scanning training [4, 5], limb activation [6], sustained attentional training [7], neck muscle vibration [8, 9], and trunk rotation [10].

While monitoring of all activities of a patient is very difficult, monitoring a specific activity of a patient preciselyis possible. Yilmaz [11] reports the monitoring of epilepsy in the brain of a patient with a wireless system. Another monitoring system [12] in the form of a wrist-worn device transmits patients information e.g. R-wave to a permanent record or to a caregiver. A wireless colonoscopy [13] is another monitoring device that transmits the pictures that it takes through its travel in the colon to a recorder worn on the belt. Orand [14] also applied a wireless monitoring system for alarming patients with inattention disorder to assist them in their transfer from wheelchairs to beds. Many more researches [15-19] deal with the wireless monitoring of the patients, elderly and the physically challenged individuals. The majority of these systems only deal with the monitoring of the status of a subject using a computer.

Our system not only deals with the monitoring of the subjects but it also has the capability of actuating a certain task. It can be expanded to includ more nodes for monitoring and for actuating more tasks. Unlike the other sytems, we have shown how to directly interface the circuitries to a wireless monitoring system to actuate a certain task. The interface and application of a microprocessor with aXbee module is also 
discussed. Another advantage of our system compared to some of the referenced systems is its affordability and simplicity.

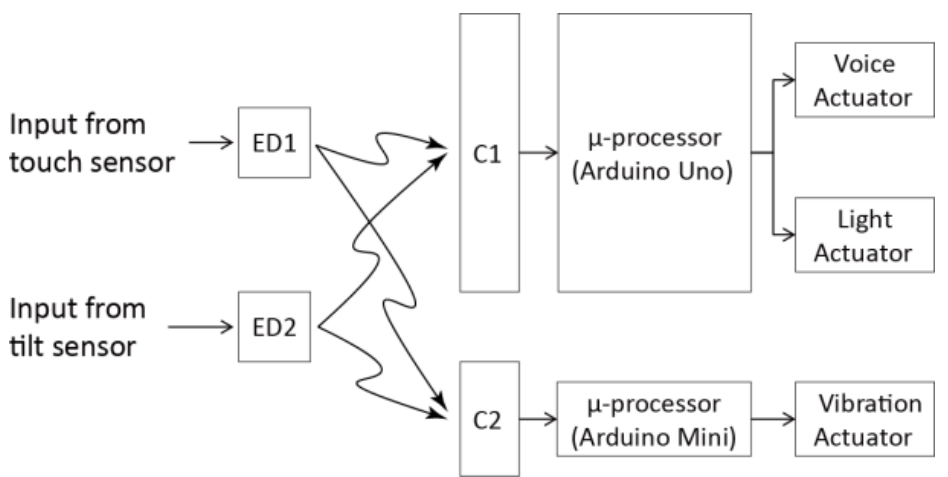

(a)

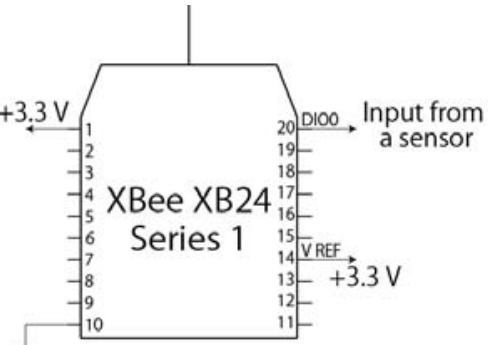

(b)

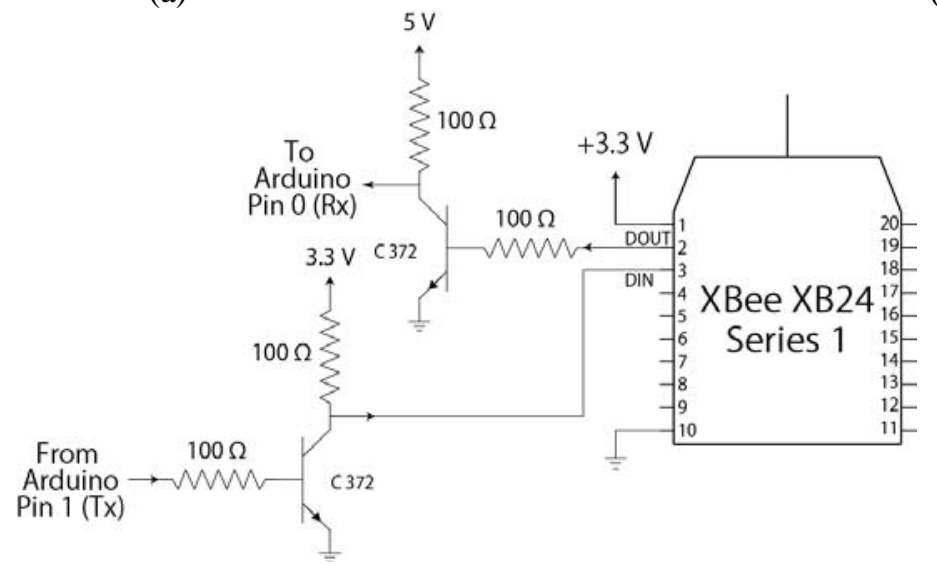

(c)

Figure 1. A: The whole system’s lay-out, B: End Device XBee modules circuitry, C: Coordinator XBee module circuitry

\section{DESIGN AND IMPLEMENTATION}

The whole system lay-out is shown in Figure 1A. The system consists of two parts, End Devices (EDs) and Coordinator. Both ED and Coordinator are connected to Series 1 XBee (Digi International Inc.) modules which are shown in Figures $1 \mathrm{~A}$ and B, respectively. While the ED transfers the feedback from the user (inputs from touch and tilt sensors) to the Coordinator in a wireless manner, the Coordinator collects the feedback information and passes it to an Arduino Uno or Mini (Arduino) microprocessor for further analysis and stimulation. Both the light and voice alarming stimuli can be placed at one place or be mounted on a wheelchair for example; therefore one coordinator and one microprocessor can be used for the actuation of both stimuli.

Since the vibrating stimulus is used directly on the patient, the stimulus requires its own ED XBee module and microprocessor (Mini). The flow chart of Figure 2 illustrates the steps of the algorithm used for the process of ED information at the Coordinator microprocessor part. ED section of this multimodal- input and output system consists of two sensors, touch and tilt, each of which is connected to an XBee module. The Coordinator section is responsible foractuating a certain task such as triggering a vibrating motor.

\subsection{ED Section}

The ED devices sections consist of two sensors, touch and tilt. The circuitries of the two sensors are shown in Figure 3. The outputs of the two circuitries are connected to the Pin 20 (DIO0) of End XBee modules.

\section{Touch Sensor}

The schematic diagram of touch sensor is shown in Figure 3A. A Sensatec HTSW (SENSATEC) touch sensor was used to find whether the hand of the patient has reached the bedrail or not. The signal from 
this sensor was amplified by 3 times and was fed into a transistor which worked as a switch. The output voltage of the transistor (3.3 or 0 volts) was fed into the Pin 20 (DIO 0) of an ED XBee module. The status of Pin 20 was sent to the coordinator XBee module in a wireless manner.

\section{Tilt Sensor}

Figure 3B shows the circuitry diagram of the tilt sensor. It consists of a tilt sensor (RBS32 0100, ONCWUE Co.), some resistors and a switching transistor. The tilt switch was used to find the angle status of the brake lever. The transistor provided the Pin 20 (DIO 0) of an ED XBee module with appropriate input of either 3.3 or 0 volts whose status was sent to the coordinator XBee module in a wireless manner.

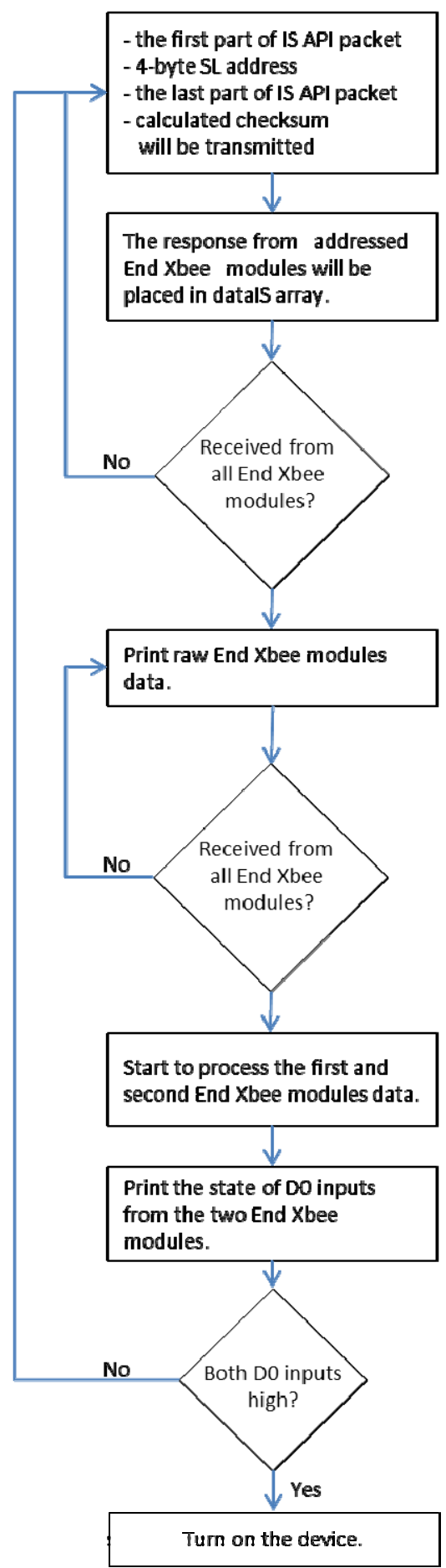

Figure 2. The flow chart of the applied algorithm in the microprocessor connected to the Coordinator XBee is shown 


\subsection{ED XBee Modules}

The ED XBee modules were set to communicate with the Coordinator XBee module in an Application Programming Interface (API) packet method. Using this method, ED XBee modules are controlled and information transformed to and from them using AT commands. Two ED XBee modules (End 1 and End 2) were used for getting the statuses of the two sensors, touch and tilt. Both XBee modules parameters were set according to the setting shown in Table 1. XBee adapter kit AE-XBee-REG-DIP was used with the two XBee.

Table 1. ED XBee modules setting

\begin{tabular}{ll}
\hline \multicolumn{1}{c}{ Parameter } & \\
\hline CE (Coordinator Enable) & $=0 \times 1 F F F$ \\
SC (Scan Channels) & $=$ Preferably 3 or 4 characters to name the end device \\
NI (Node Identifier) & $=1-$ API ENABLED \\
AP (API Enable) & $=6-0110 \mathrm{~B}$ \\
A1 (End Device Association) & $=2$ \\
RN (Random Delay Slots) &
\end{tabular}

Table 2. Coordinator XBee module setting

\begin{tabular}{lll}
\hline \multicolumn{1}{c}{ Parameter } & & Setting \\
\hline MY (16-Bit Source Address) & $=5678$ \\
CE (Coordinator Enable) & $=1-$ COORDINATOR \\
SC (Scan Channels) & $=0 x 1 \mathrm{FFE}$ \\
NI (Node Identifier) & $=$ Preferably 3 or 4 characters to name the end device \\
AP (API Enable) & $=1-$ API ENABLED \\
A2 (Coordinator Association) & $=6-0110 \mathrm{~B}$ \\
\hline
\end{tabular}

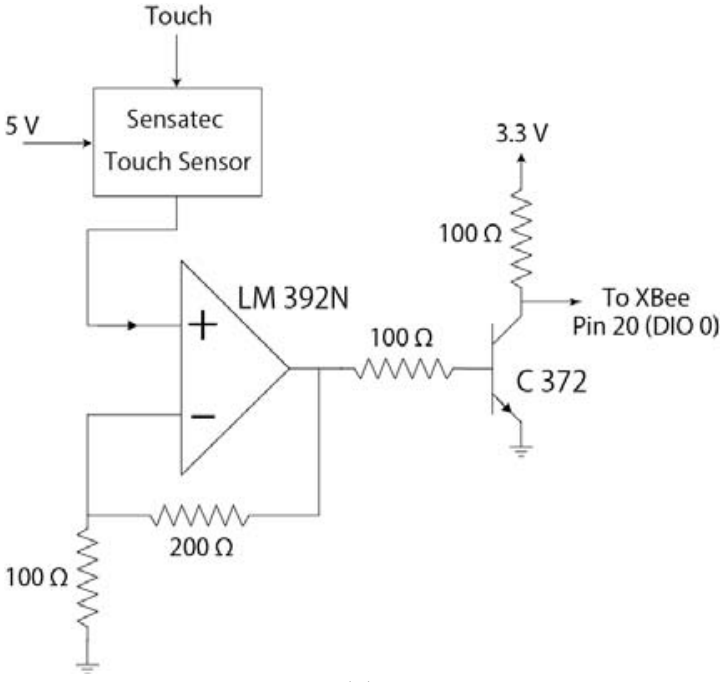

(a)

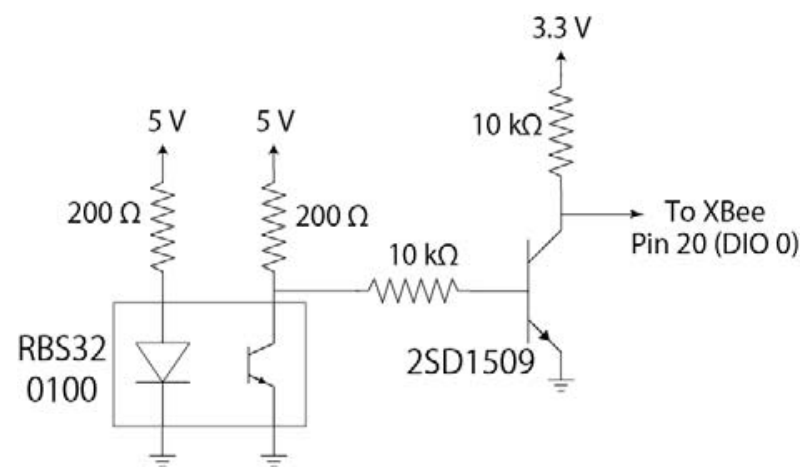

(b)

Figure 3. A: Touch sensor circuitry, B: Tilt sensor circuitry

\subsection{Coordinator Section}

The coordinator section consists of a coordinating XBee module whose Pins 2 and 3 (DOUT and DIN) were connected to Pins 0 and 1 (Rx and Tx) of an Arduino microprocessor. For voice playing and light illumination actuation, an Arduino Uno was used. Arduino Mini was used for vibration actuation. 


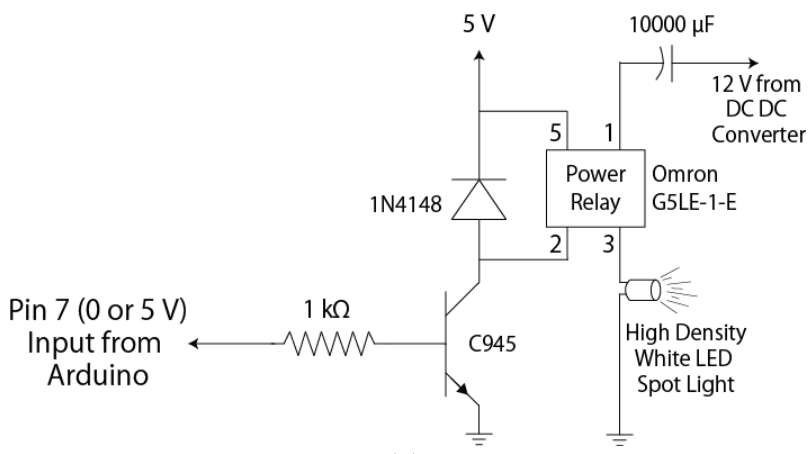

(a)

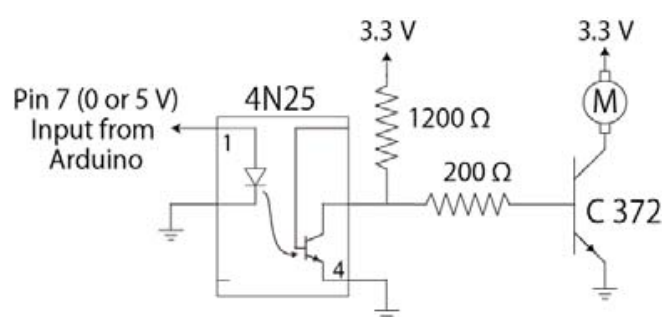

(b)

Figure 4. A: Light illumination circuitry, B: Vibrator circuitry

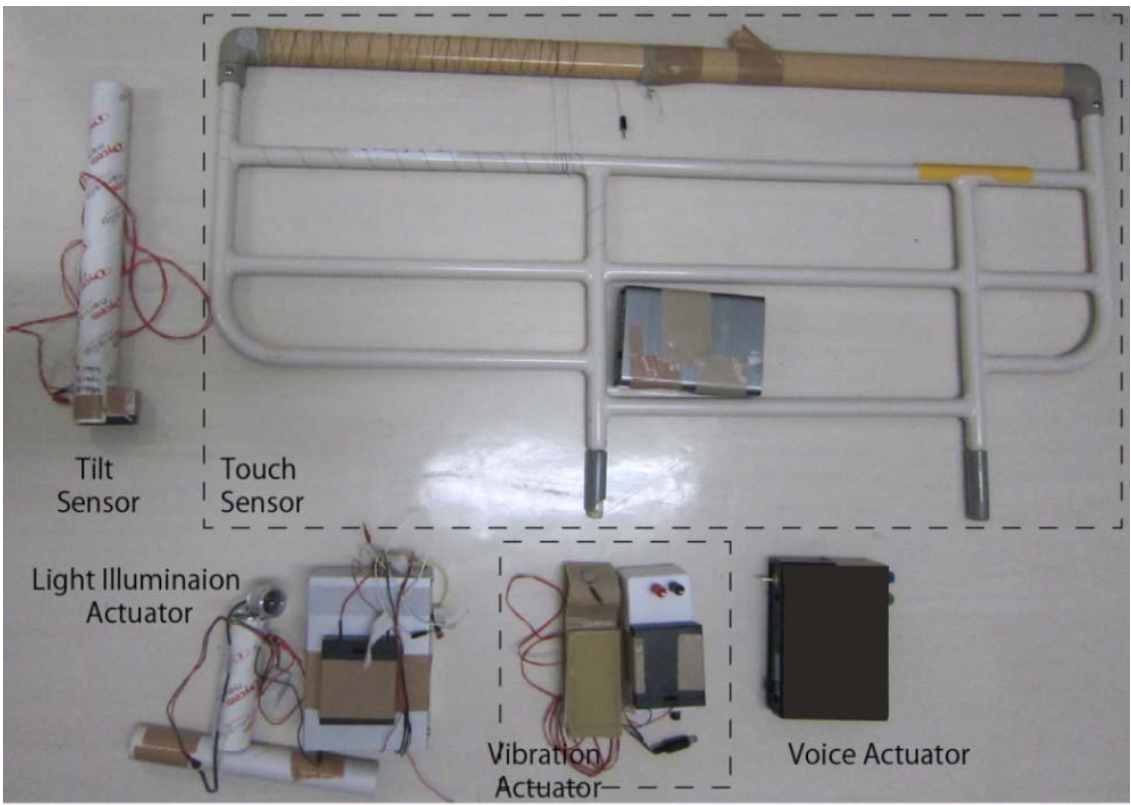

Figure 5. The multi-inputs and outputs system is shown

The touch sensor consists of a bed rail with a very thin copper wire twisted around its area where the patient usually grabs for transforming to his bed. The tilt sensor is made out of a paper core pipe with a tilt sensor on one of its ends. The other end of the pipe is fixed on the lever of the brake. The light alarming stimuli device consists of a single box (the circuitry together with a microprocessor and batteries boxes) and the lighting part (paper core pipe and a high intensity white LED). The light part was mounted on the front of the wheelchair. Vibration actuator has two parts. The circuitry together with a microprocessor and battery boxes are attached together as a single box. The other part is the vibrating motor together with its garment band. The circuitry together with a microprocessor and an rMP3 are embedded in the black box shown. A speaker is also used which is not shown in the picture.

\section{Voice Player Actuation}

An Arduino Uno module for communicating with an external device via a UART using C language, an rMP3 - Playback module shield (ROGUE robotics), and a speaker were used for voice playing. The shield's C language programs and firmware for communicating with Arduino can be downloaded from Rogue Robotics Open Source Code. Pins 0 and 1 of the Arduino (Rx and Tx) were connected to the outputs of the Coordinator XBee as it is shown in Figure 1B.

\section{Light Illumination Actuation}

Figure 4A shows the circuitry of the light sensor. A transistor, a small signal fast switching diode, a resistor, a power relay (G5LE-1-E, Omron), a dc-dc converter (UP2577ADJ-12 V), an electrolytic capacitor, and a high intensity white LED spot light were used in this circuitry. The transistor was used as a switch. The power relay and diode were used to isolate the microprocessor from high voltage. 


\section{Vibration Actuation}

The vibrator circuitry is shown in Figure 4B. A photocoupler, two resistors, a transistor, and a coin vibration motor were used as the components of this actuator. The photocoupler isolated the microprocessors from the motor's back electromotive force.

\section{Coordinator XBee Module}

The coordinating XBee module's parameters were set according to the settings shown in Table 2. The circuitry for the module is shown in Figure 1C. Two transistors were used to change the amplitude of the voltages from DOUT and to DIN to respective 5 and 3.3 volts. The microprocessor connected to the Coordinator XBee module executed the algorithm [20] shown in Figure 2. The implemented C language of the algorithm is provided in the Appendix. Based on the requirement of the patient, the program was modified.

\section{DISCUSSION}

Through this research, we could design and develope a system (Figure 5) that can be used as a base for multimodal- inputs and outputs of a monitoring-actuating system. Using API methodology, an expandable wireless network between two ED modules and one Coordinator module is purposed. By minimum modification of $C$ language program of the microprocessor and the addition of another ED XBee module, the system can be easily expanded to include more ED sensors e.g. a sensor that shows the status of the location of the patient compared to other researches [21] in which the embedment of sensors are not easy.Beforehand mentioned researches apply pervasive sensors for building smart environments to observe the activitie of daily living of patients or the elderly.

The circuitries of sensors are discussed in detail. By minimum number of electrical components, it is shown how to implement circuitries for different types of sensors. The components used in the circuitries are not expensive and allow affordable construction of portable and small devices. For example, transistors are used for both as a switching part and also as a converting component (from $5 \mathrm{~V}$ to $3.3 \mathrm{~V}$ and backward). For the isolation of sensitive components such as microprocessor, optocouplersand relay switches are used.

\section{CONCLUSION}

A wireless multi- input output system is proposed and discussed in detail. The circuitries of sensors are also presented and discussed. For both, the communication and the circuitries sections, affordable and available parts are used that makes the whole system easily implementable for situations where wireless communication is essential.

\section{ACKNOWLEDGEMENTS}

The authors have no competing interests. This study and the procedure of the experiment were approved by the local Ethics Committee of the Nanakuri Sanatorium of Fujita Health University where the study took place.

\section{REFERENCES}

[1] A. Goel, et al., "A Novel Low Noise High Gain CMOS Instrumentation Amplifier for Biomedical Applications", International Journal of Electrical and Computer Engineering (IJECE), vol. 3, no. 4, pp. 516 523, August 2013.

[2] A. Rizal, et al., "Development of Wireless Patient's Vital Sign Monitor Using Wireless LAN (IEEE.802.11.b/g) Protocol”, International Journal of Electrical and Computer Engineering (IJECE), vol. 4, no. 6, pp. 893 901, December 2014.

[3] S. Cappa, et al., "Remission of hemineglect and anosognosia during vestibular stimulation", Neuropsychologia, vol. 25, pp. 775-782, 1987.

[4] R. Luukkainen-Markkula, et al., "Rehabilitation of hemispatial neglect: a randomized study using either arm activation or visual scanning training”, RestorNeurolNeurosci, vol. 27, pp. 663-672, 2009.

[5] K. Polanowsak, et al., "Left-hand somatosensory stimulation combinedwith visual scanning training in rehabilitation for post-stroke hemineglect: a randomized, double-blind study”, Neuropsychol Rehabil, vol. 19(3), pp. 364-382, 2009.

[6] J.S. Sulzer, et al., "Preswing knee flexion assistance is coupled with hip abduction in people with stiff-knee gait after stroke”, Stroke, vol. 41, pp. 1709-1714, 2010.

[7] I.H. Robertson, et al., "Sustained attention training for unilateral neglect: theoretical and rehabilitation implications”, J Clin Exp Enuropsychol, vol. 17: pp. 416-430, 1995. 
[8] B. Biguer, et al., "Neck muscle vibration modifies the representation of visualmotion and direction in man", Brain, vol. 11, pp. 1405-1424, 1988.

[9] J.L. Taylor, D.I. McCloskey, "Illusions of head and visual target displacement induced by vibration of neck muscles”, Brain, vol. 114, pp. 755-759, 1991.

[10] H.O. Karnath, K. Christ, W. Hartje, "Decrease of contralateral neglect by neck muscle vibration and spatialorientation of trunk midline”, Brain, vol. 116, pp. 383-396, 1993.

[11] Y. Gürkan, "Wireless Power Transfer and Data Communication for Intracranial Neural Implants Case Study: Epilepsy Monitoring”, Thèse Écolepoly technique fédérale de Lausanne EPFL, 2014.

[12] T.F. Budinger, "Biomonitoring with wireless communications", Annual Review of Biomedical Engineering, vol. 5, pp. 383-412, 2013.

[13] M. Appleyard, et al., "Wireless-capsule diagnostic endoscopy for recurrent small-bowel bleeding”, New England Journal of Medicine, vol. 344(3), pp. 232-233, 2001.

[14] A. Orand, et al., "Multi-input/output alarming system for patients with inattention caused by higher cortical function disorder”, BioMedical Engineering On Line, vol. 12, 12, pp. 104, 2013.

[15] B. Zhou, et al., "A Wireless Sensor Network for Pervasive Medical Supervision”, Proceedings of the 2007 IEEE International Conference on Integration Technology, Shenzhen China, March 2007.

[16] P.S. Pandian, et al., "Smart Vest: Wearable multi-parameter remote physiological monitoring system”, Medical Engineering and Physics, vol. 30, pp. 466-477, 2008.

[17] Y.D. Lee, W.Y. Chung, et al., "Wireless sensor network based wearable smart shirt for ubiquitous health and activity monitoring”, Sensors and Actuators, vol. B 140, pp. 390-395, 2009.

[18] S.W. Lee, et al., “A Remote Behavioral Monitoring System for Elders Living Alone”, International Conference on Control, Automation and Systems, Seoul Korea, October 2007.

[19] M. Kaczmarek, et al., "Multimodal platform for continuous monitoring of elderly and disabled", Proceedings of the Federated Conference on Computer Science and Information Systems, pp. 393-400, 2011.

[20] J. Titus, “THE HANDS-ON XBee LAB MANUAL: Experiments that Teach you XBee Wireless Communication”, ELSEVIER, New York, 2012.

[21] L. Atallah, et al., "Wirelessly Accessible Sensor Populations (WASP)for Elderly Care Monitoring", the 2nd International Conference on Pervasive Computing Technologies for Healthcare, Tampere, pp. 2-7, 2008.

\section{APPENDIX}

I*

Wireless XBee Series 1 Network

*I

I/

//Create start-of-API packet for IS command with this array of bytes.

//Byte count $0 \times 0 \mathrm{~F}$ does not include first 3 bytes or the checksum byte.

byte packetIS_start] $=\{0 \times 7 \mathrm{E}, 0 \times 00,0 \times 0 \mathrm{~F}, 0 \times 17,0 \times 52,0 \times 00,0 \times 13,0 \times A 2,0 \times 00\}$;

intpacketlS_start_len $=9$;

//Create end-of-API packet for IS command

bytepacketIS_end $]=\{0 \times \mathrm{FF}, 0 \times \mathrm{FE}, 0 \times 02,0 \times 49,0 \times 53\}$;

intpacketlS_end_len $=5$;

//Define number of modules here as a constant

constintnumb_of_modules = 2;

//Define number of bytes in arrays as constants

constintdatalslength $=40$;

constintdataNDlength $=40$;

//Set up array with End-device 64-bit address starting at dataND[x][7]

//remember, dataND[] array starts with dataND[x][0]

//array definitions require a constant value

bytedataND[numb_of_modules][dataNDlength] $=\{$

$\{0 \times 00,0 \times 00,0 \times 00,0 \times 00,0 \times 00,0 \times 00,0 \times 00$,

$0 \times 00,0 \times 13,0 \times A 2,0 \times 00,0 \times 40,0 \times 8 A, 0 \times 95$,

$0 \times 13,0 \times 00,0 \times 41,0 \times 41,0 \times 41,0 \times 41,0 \times 00\}$,

$\{0 \times 00,0 \times 00,0 \times 00,0 \times 00,0 \times 00,0 \times 00,0 \times 00$,

$0 \times 00,0 \times 13,0 \times A 2,0 \times 00,0 \times 40,0 \times 89,0 \times 3 E$,

$0 \times C 7,0 \times 00,0 \times 42,0 \times 42,0 \times 42,0 \times 42,0 \times 00\}$,

\} ;

//Create an array for the response from each End-device module

bytedatalS[numb_of_modules][datalSlength];

//Define bit masks for expected digital bits at DIOO and DIO2 pins

byte D0 mask $=0 \times 01 ; \quad / / 00000001$

byte D2_mask = 0x04; // 00000100

//Define ADC reference-voltage input, change as needed.

floatVref = 3.3;

constintdigitalOutPin = 7; // digital output pin to turn the alarming system ON and OFF 


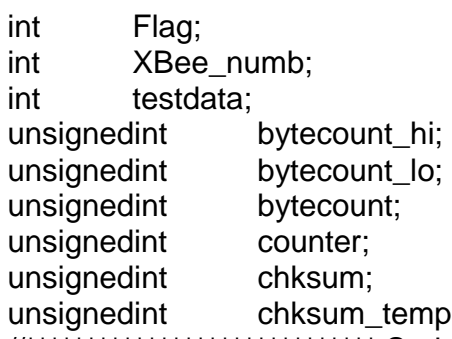

//Checksum is now calculated and sent. Using AND operator (\&), save only eight least-//significant bits for subtraction 
Serial.write(0xFF - (chksum\& 0xFF));

//Get responses from End XBee modules via the serial port of Arduion

testdata $=$ Seriallnput () ;

if (testdata $==0 \times 7 \mathrm{E})$;

//Start delimiter of a frame (0x7E) //if yes

//Get the two high and low bytes of the sensor reading

bytecount_hi $=$ Seriallnput();

bytecount $10=$ Seriallnput();

bytecount $=$ (bytecount_hi * 256) + bytecount_lo; //the number of bytes in the message

chksum temp $=0$; $\quad$ //clear checksum value

for (counter = 0; counter <bytecount; counter++) I/put End XBee data in an array

datalS[XBee_numb][counter] $=$ Seriallnput $($ )

chksum_temp = chksum_temp + datalS[XBee_numb][counter];

\}

chksum = Seriallnput(); $\quad$ //get checksum--last byte

//1 End XBee data was obtained, do it //for the $2^{\text {nd }}$ one

//The data for both2 End XBee modules was obtained.

XBee_numb $=0$;

//Processing the digital data from both 2 End XBee modules.

for (XBee_numb $=0$; XBee_numb<numb_of_modules; XBee_numb++)

//Check the pin D0 statuses of the 2 End XBee modules and turn on the alarming system if //both End XBee pins are high switch (XBee_numb)

\{

case 0:

\{

if ((datalS[XBee numb][19] \& Do mask $)>0)$

\{

Flag=1;

\}

else

Flag $=0$

digitalWrite(digitalOutPin,LOW):

\}

break;

\}

case 1:

if $($ Flag $==1)$

\{

if $(($ datalS[XBee_numb][19] \& D0_mask $)>0)$

digitalWrite(digitalOutPin,HIGH); // set the output pin status to LOW

\}

else

\{

digitalWrite(digitalOutPin,LOW)

break;

\}

while(0) \{\}$\quad$ //do-nothing statement

//this part can be used to turn the alarming system on or off for a certain amount of time while(1)

\{

break;

\}

\} $\quad$ //the end of the main loop()

On the Design and Development of a Zigbee-Based Multimodal Input-Output Monitoring-... (A. Orand) 


\section{BIOGRAPHIES OF AUTHORS}
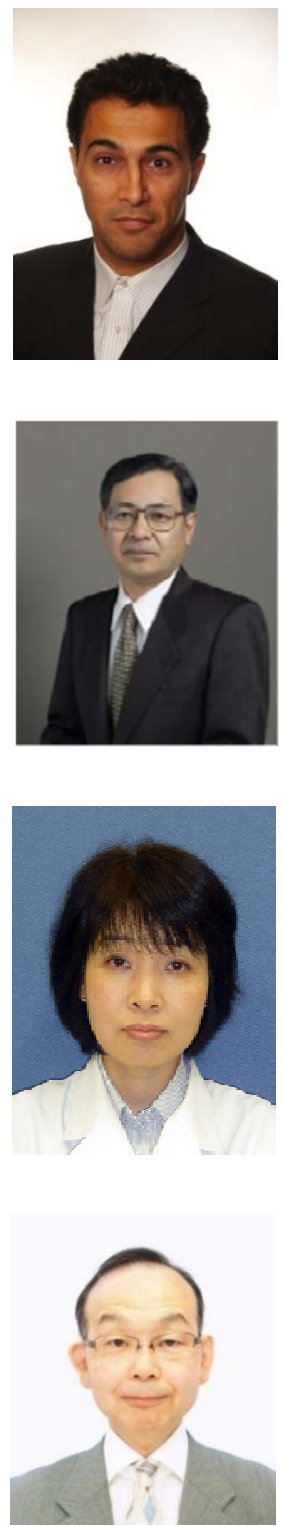

Abbas Orand obtained his B.Sc. and M. Sc. degrees in electrical and mechatronics engineerings from Dalhousie University in Canada and Siegen University in Germany in 2003 and 2008, respectively. From 2007 to 2008, he worked at Daimler AG in Germany as a researcher. He obtained his doctor of philosophy degree in the field of informatics and biosciences from Keio University in Japan in 2012. He is with the Rehabilitation Department of Fujita Memorial Nanakuri Institute at Fujita Health University since 2011. His fields of research interests are robotics, neuromuscular electrical stimulation, biomedical engineering and rehabilitation.

Yutaka Tomita received a Bachelor of Engineering, a Master of Engineering, and a Doctor of Engineering from Keio University, in 1973, 1975, and 1982, respectively. He also received a Doctor of Medical Science from Kitasato University in 1994. He was a research associate in School of Medicine, Keio University, an assistant professor, an associate professor and a professor in Faculty of Science and Technology, Keio University until 2010. He was a professor in Fujita Memorial Nanakuri Institute, Fujita Health University during 2011 and 2015. His fields of research interest are electrical physiology, biomedical measurement, clinical engineering, etc. He is a member of IEEE.

Sayaka Okamoto received her Doctor of Medicine from Mie University in 1993. She received her Doctor of Philosophy from Fujita Health University in 2011. At the moment, she is an assistant professor at the Department of Rehabilitation Medicine II, Shool of Medicine, Fujita Health University. Her current research topic is the rehabilitation of stroke patients.

Sonoda Shiger received his Doctors of Medicine and of Philosophy from Keio University in 1985 and 1995, respectively. From 1997 to 2000, he was an assistant professor at the Department of Rehabilitation Medicine of the School of Medicine, Keio Univrsity. He worked respectively as an associate professor and a professor between the years of 2000-2002 and 2002-2007 at the Department of Rehabilitation Medicine of the School of Medicine of Fujita Health University. From 2003, he has served as the president of the Nanakuri Sanatorium of Fujita Health University. He has been the professor and chairman of the Department of Rehabilitation Medicine II of the School of Medicine of Fujita Health University since 2007. 